\title{
Анизотропия отрицательного магнетосопротивления в эапитаксиальных слоях GaMnAs
}

\author{
(C) A.С. Газизулина ${ }^{1}$, А.А. Насиров ${ }^{1}$, А.А. Небесный ${ }^{1}$, П.Б. Парчинский ${ }^{1, \uparrow}$, Dojin Kim $^{2}$ \\ ${ }^{1}$ Национальный университет Узбекистана, \\ 100176 Ташкент, Узбекистан \\ ${ }^{2}$ Chungnam National University, \\ 305-764, Taejon, Korea \\ ฯ E-mail: pavelphys@mail.ru
}

Поступила в Редакцию 10 августа 2020 г.

В окончательной редакции 22 октября 2020 г.

Принята к публикации 22 октября 2020 г.

Исследована температурная зависимость анизотропии магнитотранспортных свойств эпитаксиальных слоев GaMnAs, находящихся в состоянии ферромагнитного упорядочения. Показано, что в исследуемых слоях наблюдается анизотропия отрицательного магнетосопротивления, не связанная с наличием одноосной анизотропии и ориентацией оси жесткого намагничивания. Данная анизотропия может быть связана с наличием в слое GaMnAs пространственно-ориентированных структур, возникающих в объеме эпитаксиального слоя в процессе его роста.

Ключевые слова: анизотропия, отрицательное магнетосопротивление, эпитаксиальные слои, ферромагнитное упорядочение.

DOI: 10.21883/FTP.2021.02.50503.9502

\section{1. Введение}

Полупроводниковая спинтроника является одной из наиболее быстро развивающихся областей микро- и наноэлектроники. Основная концепция спинтроники состоит в том, что для передачи информации используется как заряд носителей, так и их спиновая ориентация.

Реализация приборов и структур полупроводниковой спинтроники требует создания новых материалов, обладающих сочетанием полупроводниковых и магнитных свойств. Обнаружение ферромагнетизма в арсениде индия, легированного марганцем, вызвало интерес к разбавленным магнитным полупроводникам (РМП) на основе соединений $\mathrm{A}^{\mathrm{III}} \mathrm{B}^{\mathrm{V}}$. На сегодняшний день такого рода РМП рассматриваются в качестве основных материалов полупроводниковой спинтроники [1-3]. Особое место среди этих материалов занимает GaMnAs. Перспективность применения GaMnAs обусловлена его структурной совместимостью с широким классом полупроводниковых приборов и структур на основе GaAs, a также возможностью интеграции технологического режима его получения с технологией получения традиционных, немагнитных полупроводниковых структур.

Согласно принятым на сегодняшний день моделям, ферромагнитное упорядочение в РМП возникает в результате взаимодействия между локализованными спинами магнитных ионов и делокализованными или слаболокализованными дырками [4,5]. B GaMnAs атомы марганца, занимая позиции в подрешетке галлия, являются носителями локализованных спинов и выступают одновременно как акцепторы, поставляющие дырки в валентную зону полупроводника, что исключает необходимость его легирования дополнительными примеся- ми $[4,6]$. В то же время концентрация магнитных ионов, необходимая для создания состояния ферромагнитного упорядочения в полупроводниковой матрице $\mathrm{GaAs}$, coставляет $10^{17}-10^{18} \mathrm{~cm}^{-3}$, что значительно превышает предел растворимости Mn в GaAs. Поэтому GaMnAs c концентрацией $\mathrm{Mn}$, достаточной для создания состояния ферромагнитного упорядочения, может быть получен только при помощи физически неравновесных методов, таких как низкотемпературная молекулярнолучевая эпитаксия (НТ МЛЭ) [7,8]. Однако данный метод обладает существенным недостатком, а именно низкая температура эпитаксии является причиной низкого кристаллического качества и высокой концентрации дефектов в выращиваемых эпитаксиальных слоях $[9,10]$.

Кроме того, содержание значительной концентрации примеси $\mathrm{Mn}$ в полупроводниковой матрице приводит к тому, что постоянная решетки GaMnAs становится больше, чем постоянная решетки нелегированного марганцем арсенида галлия. Повышенная дефектность слоев GaMnAs и несоответствие параметров решетки GaMnAs и подложки GaAs приводит к тому, что в растущем эпитаксиальном слое возникают поля деформаций и механических напряжений. Наличие деформаций в свою очередь приводит к изменению порядка симметрии GaMnAs и сопровождается изменением его зонной структуры, вследствие чего магнитные и транспортные характеристики эпитаксиального слоя GaMnAs отличаются от характеристик ненапряженного кристалла. Одним из важнейших следствий понижения симметрии в эпитаксиальных слоях GaMnAs является изменение параметров анизотропии его магнитных свойств, таких как магнитная восприимчивость, магнитный момент и ориентация оси легкого намагничивания. 
К настоящему времени установлено, что характер эффектов, связанных с анизотропией магнитных свойств РМП на основе GaAs, зависит от величины и знака механических напряжений в эпитаксиальном слое, температуры, а также концентрации носителей заряда в его объеме [11-14]. В то же время природа этих зависимостей не может считаться окончательно установленной. Поскольку анизотропия магнитных свойств GaMnAs может оказывать определяющее влияние на параметры создаваемых на основе GaMnAs приборов и структур, исследование магнитной анизотропии эпитаксиальных слоев GaMnAs представляет значительный интерес.

Наличие корреляции между магнитными и транспортными свойствами разбавленных магнитных полупроводников позволяет проводить исследования анизотропии их магнитных свойств при помощи достаточно простых магнитотранспортных измерений $[11,15]$. В настоящей работе приводятся результаты исследования анизотропии магнитных свойств эпитаксиальных слоев GaMnAs, полученные посредством измерения его магнетосопротивления.

\section{2. Образцы и методика эксперимента}

Исследуемые образцы были получены НТ МЛЭ на полуизолирующую подложку GaAs с кристаллографической ориентацией (001). Перед выращиванием эпитаксиального слоя GaMnAs выращивался буферный слой GaAs при температуре подложки $450-500^{\circ} \mathrm{C}$. После этого температура подложки опускалась до $275^{\circ} \mathrm{C}$ для выращивания эпитаксиального слоя GaMnAs. Контроль структурной однородности эпитаксиального слоя в процессе его роста осуществлялся при помощи дифрактометра отражённых быстрых электронов. Температура эффузионной ячейки $\mathrm{Mn}$ в процессе выращивания составляла $860^{\circ} \mathrm{C}$. Данные условия позволили получить однородные эпитаксиальные слои GaMnAs с концентрацией примеси $\mathrm{Mn} 0.78$ aт\% (концентрация примеси $\mathrm{Mn}$ в исследуемых образцах определялась с помощью электронно-зондового микроанализа). Толщина исследуемых образцов составляла 250-300 нм. Исследования, проведенные с помощью рентгеноструктурного анализа, также подтвердили, что образцы являются однородными и не содержат включений других фаз. Концентрация основных носителей заряда, определенная при комнатной температуре из измерений эффекта Холла, составила $7 \cdot 10^{18} \mathrm{~cm}^{-3}$. Температура Кюри исследуемых образцов была определена из измерений температурной зависимости аномального эффекта Холла и составила $50-55 \mathrm{~K}[16]$.

Для исследования анизотропии магнитных свойств эпитаксиальных слоев GaMnAs в настоящей работе проводились измерения магнетосопротивления вдоль различных кристаллографических направлений. Измерения проводились четырехзондовым методом в геометрии
Ван-дер-Пау. В процессе измерений магнитное поле было ориентировано нормально к поверхности образца.

\section{3. Результаты и обсуждение}

На рис. 1 представлены результаты измерений магнетосопротивления, полученные для различных кристаллических осей при температуре $20 \mathrm{~K}$. Для удобства представления результатов зависимости магнетосопротивления от $H$ представлялись в нормированном виде

$$
R_{h}=\frac{R(H)-R(0)}{R(0)},
$$

где $R(H)$ - сопротивление, измеренное при заданном значении магнитного поля, $R(0)$ - сопротивление образца в отсутствие магнитного поля.

Обращает на себя внимание, что в случае оси [110], в области полей до 4000 Э наблюдается положительное магнетосопротивление, достигающее своего максимума при значениях $H=2000$ Э. В случае осей [110], [100] и [010] положительное магнетосопротивление отсутствует, однако в области полей до 2500 Эаблюдается плато магнетосопротивления. Наибольшая ширина плато, до 2500 Э, наблюдается для осей $[010]$ и [100], а наименьшее, до $1000 Э$, - для оси [110]. Отметим, что начиная с некоторого значения напряженности магнитного поля $H_{t}$ величина $R_{h}$ зависит от $H$ практически линейно. Величина $H_{t}$ различна для различных кристаллографических направлений. Наибольшее значение $H_{t}=4000$ Э наблюдается для значений $R_{h}$, измеренных вдоль оси [110], а наименьшее $H_{t}=1500$ Э - для значений $R_{h}$, измеренных вдоль оси [110].

Согласно существующим моделям в разбавленных магнитных полупроводниках, находящихся в состоянии ферромагнитного упорядочения, магнетосопротивление

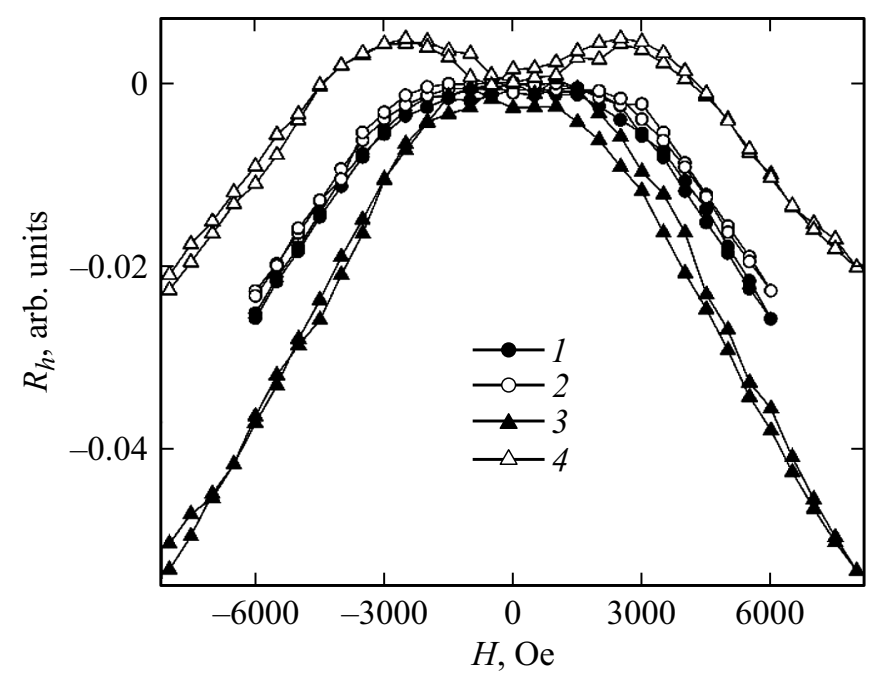

Рис. 1. Магнетосопротивление GaMnAs, измеренное вдоль различных кристаллических осей при температуре $20 \mathrm{~K}$ : $1-[010], 2-[100], 3-[110], 4-[1 \overline{1} 0]$. 
в области сильных и слабых полей определяется различными механизмами. В области слабых полей основной вклад в величину магнетосопротивления вносят процессы, связанные с переориентацией намагниченности образца, которые в случае перпендикулярной ориентации магнитного поля по отношению к току, протекающему через образец, приводят к возникновению положительного магнетосопротивления. В области сильных полей, когда переориентация намагниченности завершена, доминирующими становятся процессы, связанные с изменением степени локализации носителей заряда, которые обусловливают возникновение отрицательного магнетосопротивления [8]. Таким образом, наличие положительного магнетосопротивления вдоль

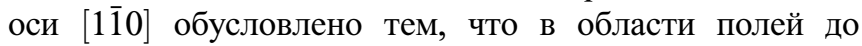
2000 Эоминирующим механизмом, определяющим характер магнетосопротивления в направлении данной оси, является рассеяние носителей заряда на разупорядоченных магнитных моментах ионов магнитной примеси $\mathrm{Mn}$.

Наличие плато магнетосопротивления, наблюдаемого для осей $[110],[100]$ и [010], свидетельствует о том, что для данных осей, в области слабых полей вклад процессов, связанных с переориентацией намагниченности образца, компенсируется вкладом процессов, связанных с изменением степени локализации носителей заряда. Линейность зависимости $R_{h}$ от $H$ при напряженностях магнитного поля, бо́льших $H_{t}$, связана с установлением магнитного упорядочения в GaMnAs, при этом вклад процессов, связанных с переориентацией намагниченности, исчезает, и величина магнетосопротивления определяется только процессами, связанными с изменением степени локализации носителей заряда.

Необходимо отметить, что в области линейной зависимости $R_{h}$ от $H$ также наблюдается анизотропия магнитных характеристик исследуемых образцов, проявляющаяся в различном наклоне зависимостей $R_{h}$ от $H$, определенных для различных кристаллических направлений. Для характеризации магнетосопротивления в области полей, больших чем $H_{t}$, мы использовали величину $R_{x}$, определяемую как

$$
R_{x}=\frac{\left(R\left(H_{\max }\right)-R\left(H_{t}\right)\right)}{H_{\max }-H_{t}},
$$

где $H_{\max }-$ максимальное значение $H$ в процессе измерений, $R\left(H_{\max }\right)$ и $R\left(H_{t}\right)$ - значения величины $R_{h}$ при напряженности магнитного поля $H_{\max }$ и $H_{t}$ соответственно.

Наибольшее значение $R_{x}=0.83 \cdot 10^{-5}$ наблюдается для оси [1ㅣㅣ, тогда как наименьшее $R_{x}=0.57 \cdot 10^{-5}-$ для оси [110]. Таким образом, можно отметить, что различие в зависимостях $R_{h}$ от $H$ наблюдается как в области слабых $\left(H<H_{t}\right)$, так и в области сильных полей $\left(H>H_{t}\right)$. Наиболее существенное различие наблюдается для осей $[110]$ и [1 10$]$, которые в ненапряженной решетке GaAs являются эквивалентными. Отметим, что различия в характере магнетосопротивления в области полей $H<H_{t}$ могут быть объяснены наличием в слое GaMnAs одноосной анизотропии, которая определяет направление осей легкого и жесткого намагничивания в эпитаксиальном слое [15,17-19]. Как следует из результатов измерений, ось жесткого намагничивания в исследуемом образце ориентирована вдоль оси [1ㅣㅣ, для которой наблюдается наибольшее значение величины $H_{t}$. Однако при значениях $H>H_{t}$, когда в объеме эпитаксиального слоя заканчивается процесс переориентации магнитных моментов и магнетосопротивление становится отрицательным, одноосная анизотропия не может рассматриваться в качестве причины различия в характере зависимостей $R_{h}$ от $H$, измеренных вдоль эквивалентных кристаллических осей.

На рис. 2 и 3 представлены температурные зависимости величин $R_{h}$ и $R_{x}$, измеренных вдоль различных кристаллических осей исследуемого эпитаксиального слоя. Наибольшие изменения в характере магнетосопротивления наблюдаются для оси [1피, для которой наибольшая величина положительного магнетосопротивления наблюдается при $20 \mathrm{~K}$. При повышении температуры абсолютная величина положительного сопротивления уменьшается, положение максимума величины $R_{h}$ смещается в сторону меньших значений $H$. Уменьшение положительного магнетосопротивления с повышением температуры связано с уменьшением степени ферромагнитного упорядочения эпитаксиального слоя GaMnAs.

При температуре $40 \mathrm{~K}$ положительное магнетосопротивление исчезает полностью, и при $H<H_{t}$ зависимости $R_{h}(H)$, полученные для оси [1ㅣㅣ, практически совпадают с аналогичными зависимостями, полученными для оси [110], что свидетельствует об исчезновении одноосной магнитной анизотропии при повышении температуры. Температурные зависимости магнетосопротивления, измеренные вдоль кристаллических осей [100] и [010] и [110] в области полей $H<H_{t}$, демонстрируют некоторое уменьшение ширины плато магнетосопротивления, что также может быть связано с уменьшением степени ферромагнитного упорядочения с повышением температуры.

В то же время необходимо отметить, что различия в угле наклона зависимостей $R_{h}(H)$ наблюдаются во всем диапазоне исследуемых температур. Более того, из представленных на рис. 3 зависимостей видно, что температурные зависимости величины $R_{x}$, определенные для кристаллических осей $[110]$ и $[1 \overline{1} 0]$, ведут себя различным образом. Полученные результаты свидетельствуют о том, что в исследуемом образце GaMnAs анизотропия магнитотранспортных свойств, определенных вдоль осей [110] и [110], сохраняется, несмотря на исчезновение одноосной магнитной анизотропии.

Рассмотрим теперь природу возникновения эффекта анизотропии отрицательного магнетосопротивления в области полей $H>H_{t}$. На наш взгляд, различная величина угла наклона зависимостей $R_{h}(H)$, наблюдаемая для эквивалентных кристаллических осей [110] 

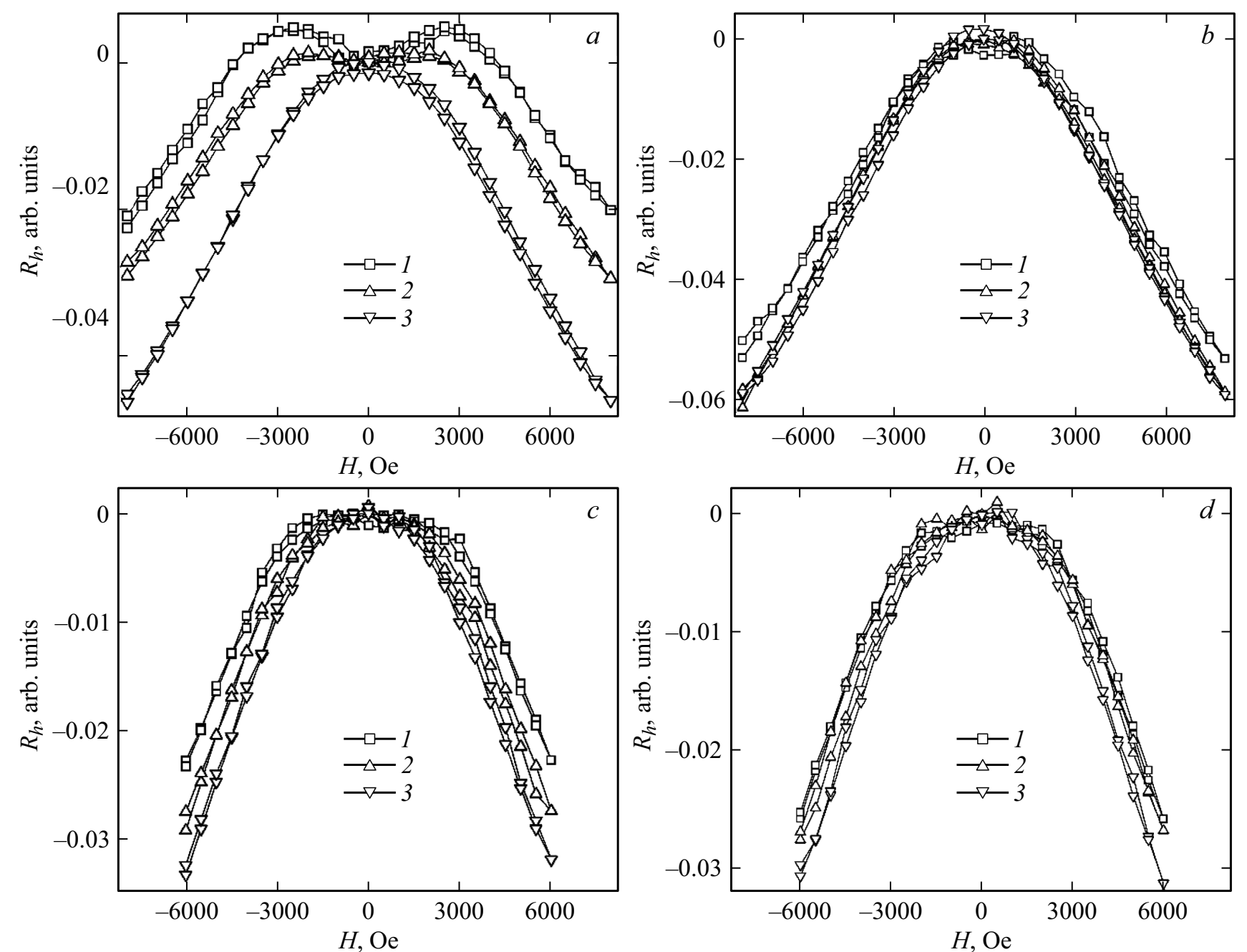

Рис. 2. Магнетосопротивление эпитаксиальных слоев GaMnAs, измеренное вдоль различных кристаллических осей при различных температурах: $a-$ вдоль оси $[1 \overline{1} 0], b-$ вдоль оси [110], $c-$ вдоль оси [100], $d-$ вдоль оси [010]. Температура измерения: К, $1-20,2-30,3-40$.

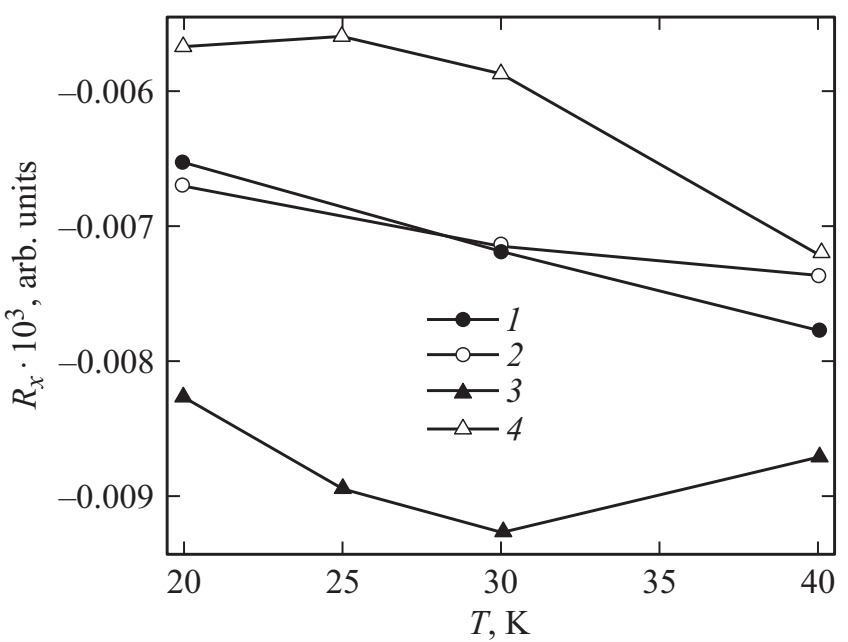

Рис. 3. Температурная зависимость угла наклона зависимостей $R_{h}(\mathrm{H})$, определенная вдоль различных кристаллических осей при значениях напряженности магнитного поля $H>H_{t}$ : $1-[010], 2-[100], 3-[110], 4-[1 \overline{1} 0]$. и [1ㅣㅣ, может быть связана с тем, что в эпитаксиальных слоях GaMnAs в процессе их роста формируются пространственно-ориентированные структуры, связанные с неоднородным распределением примеси $\mathrm{Mn}$ в полупроводниковой матрице GaAs. O возможности существования таких пространственно-ориентированных структур в эпитаксиальных слоях GaMnAs сообщается в работе [19]. Авторы работы [20] наблюдали возникновение пространственно-ориентированных структур на поверхности тонких (5-25 нм) слоев GaMnAs, выращенных на подложке GaAs при помощи НТ МЛЭ.

Природа возникновения таких пространственноориентированных структур в эпитаксиальных слоях GaMnAs к настоящему моменту остается неизученной и требует дальнейших исследований. Однако можно предположить, что эти структуры возникают на начальном этапе роста эпитаксиального слоя и связаны с характером релаксации поверхности подложки GaAs [19,21]. 


\section{4. Заключение}

В результате проведенных исследований установлено наличие анизотропии отрицательного магнетосопротивления в исследованных эпитаксиальных слоях GaMnAs, проявляющейся в различии угла наклона линейных участков зависимостей $R_{h}$ от $H$, определенных вдоль различных кристаллических осей. Показано, что наблюдаемый эффект не связан с одноосной магнитной анизотропией исследуемых образцов, а, на наш взгляд, может свидетельствовать о наличии в слоях GaMnAs пространственно-ориентированных структур, возникающих в процессе роста эпитаксиального слоя. Тот факт, что анизотропия магнитотранспортных свойств особенно ярко проявляется для осей $[110]$ и $[1 \overline{1} 0]$, на наш взгляд, свидетельствует о том, что пространственоориентированные структуры в исследованных эпитаксиальных слоях ориентированы вдоль кристаллографического направления $\langle 110\rangle$.

\section{Конфликт интересов}

Авторы заявляют, что у них нет конфликта интересов.

\section{Список литературы}

[1] Ch. Song, B. Cui, F. Li, X. Zhou, F. Pan. Progr. Mater. Sci., 87, 33 (2017).

[2] H. Munekata. In: Molecular Beam Epitaxy: Materials and Applications for Electronics and Optoelectronics, ed. by H. Asahi Y. Horikoshi (Hoboken, N.J., John Wiley \& Sons, 2019).

[3] Б.Н. Звонков, О.В. Вихрова, Ю.А. Данилов, М.В. Дорохин, А.В. Кудрин, И.Л. Калентьева, Е.А. Ларионова, В.А. Ковальский, О.А. Солтанович. ФТП, 53 (3), 351 (2019).

[4] T. Dietl, H. Ohno, F. Matsukura, J. Cibert, D. Ferrand. Scince, 287, 1019 (2000).

[5] T. Dietl, H. Ohno, F. Matsukura. Phys. Rev. B, 63, 195205-1 (2001).

[6] В.А. Иванов, Т.Г. Аминов, В.М. Новоторцев, В.Т. Калинников. Изв. АН. Сер. хим., 11, 2255 (2004).

[7] T. Hayashi, M. Tanaka, H. Shimada, N. Tsuchiya, Y. Otuka. J. Cryst. Growth, 175/176, 1063 (1997).

[8] Y. Iye, F. Oiwa, A. Endo, S. Katsumoto, F. Matsukura, A. Shen, H. Ohno, H. Munekata. Mater. Sci. Eng. B, 63, 88 (1999).

[9] A. Pross, S. Bending, K. Edmonds, R.P. Campion, C.T. Foxon, B. Gallaher. J. Appl. Phys., 95, 3325 (2004).

[10] J. Daeubler, M. Glunk, W. Schoch, W. Limmer, R. Sauer. Appl. Phys. Lett., 88, 051904 (2006).

[11] P. Juszyński, M. Gryglas-Borysiewicz, J. Szczytko, M. Tokarczyk, G. Kowalski, J. Sadowski, D. Wasik. J. Magn. Magn. Mater., 396, 48 (2015).

[12] J. Chang, S. Choi, K. Jae Lee, S.-K. Bac, S. Choi, P. Chongthanaphisut, S. Lee, X. Liu, M. Dobrowolska, J.K. Furdyna. J. Cryst. Growth, 512, 112 (2019).

[13] T. Dietl. J. Appl. Phys., 9, 7437 (2001).
[14] M. Sawicki, F. Marsukura, A. Idiaszek, T. Dietl, G.M. Schott, C. Ruester, C. Gold, G. Karczewski, G. Schmidt, L.W. Molenkamp. Phys. Rev. B, 70, 245325 (2004).

[15] K. Hamaya, T. Taniyama, Y. Kitamoto, Y. Yamazaki, R. Moriya, H. Munekata. IEEE Trans. Magnetics, 40, 2682 (2004).

[16] П.Б. Парчинский, А.С. Галашина, D. Kim. Узб. физ. журн., 19 (3), 143 (2017). (P.B. Parchinskiy, A.S. Galashina, D. Kim. Uzbek J. Phys., 19 (3), 143 (2017).

[17] S.Chung, H.C. Kim, S. Leea, X. Liu, J.K. Furdyna. Solid-State Commun., 149, 1739 (2009).

[18] V. Stanciu, P. Svedlindh. Appl. Phys. Lett., 87, 242509 (2005).

[19] U. Welp, V.K. Vlasko-Vlasov, A. Menzel, H.D. You, X. Liu, J.K. Furdyna, T. Wojtowicz. Appl. Phys. Lett., 85, 260 (2004).

[20] S. Piano, X. Marti, A.W. Rushforth, K.W. Edmonds, R.P. Campion, M. Wang, O. Caha, T.U. Schölli, V. Holý, B.L. Gallagher. Appl. Phys. Lett., 98, 152503 (2011).

[21] U. Welp, V.K. Vlasko-Vlasov, X. Liu, J.K. Furdyna, T. Wojtowicz. Phys. Rev. Lett., 90, 167206 (2003).

Редактор Г.А. Оганесян

\section{Anisotropy of negative magnetoresistance in GaMnAs epitaxial layers}

\author{
P.B. Parchinskiy ${ }^{1}$, Dojin Kim ${ }^{2}$ \\ ${ }^{1}$ National University of Uzbekistan, \\ 100176 Tashkent, Uzbekistan \\ ${ }^{2}$ Chungnam National University, \\ 305-764, Taejon, Korea
}

A.S. Gazizulina ${ }^{1}$, A.A. Nasirov ${ }^{1}$, A.A. Nebesniy ${ }^{1}$,

Abstract Anisotropy of magnetotransport properties of GaMnAs epitaxial layers in ferromagnetic state has been investigated as a function of temperature. The anisotropy of negative magnetoresistance that is not related to uniaxial anisotropy and hard axis orientation has been observed. This anisotropy may be result of arising of spatially oriented structures in the GaMnAs layer during its growth. 